This item was submitted to Loughborough's Research Repository by the author.

Items in Figshare are protected by copyright, with all rights reserved, unless otherwise indicated.

\title{
Development of event-driven optimal control for central air-conditioning systems
}

\section{PLEASE CITE THE PUBLISHED VERSION}

https://doi.org/10.1080/19401493.2020.1737231

\section{PUBLISHER}

Taylor and Francis

\section{VERSION}

AM (Accepted Manuscript)

\section{PUBLISHER STATEMENT}

This is an Accepted Manuscript of an article published by Taylor \& Francis in Journal of Building Performance Simulation on 17 Mar 2020, available online: https://doi.org/10.1080/19401493.2020.1737231

\section{LICENCE}

CC BY-NC-ND 4.0

\section{REPOSITORY RECORD}

Hou, Jin, Xiaowei Luo, Gongsheng Huang, Linfeng Zhang, Zhun Jerry Yu, and Mahroo Eftekhari. 2020. "Development of Event-driven Optimal Control for Central Air-conditioning Systems". figshare. https://hdl.handle.net/2134/11996613.v1. 


\title{
Development of event-driven optimal control for central air-
} conditioning systems

\author{
Jin HOU ${ }^{\mathrm{a}}$, Xiaowei LUO ${ }^{\mathrm{a}}$, Gongsheng HUANG ${ }^{\mathrm{a}}$, Linfeng ZHANG ${ }^{\mathrm{a}, \mathrm{b}}$, Zhun (Jerry) YU ${ }^{\mathrm{c}}$, \\ Mahroo EFTEKHARI ${ }^{\mathrm{d}}$
${ }^{a}$ Department of Architecture and Civil Engineering, City University of Hong Kong, Kowloon, Hong Kong
'Department of Underground Engineering, Southeast University, Nanjing, Juangsu, 211189, China \\ ${ }^{\mathrm{c} C o l l e g e}$ of Civil Engineering, National Center for International Research Collaboration in Building Safety and \\ Environment, Hunan University, Changsha, Hunan, 410082, China \\ ${ }^{\mathrm{d} S c h o o l}$ of Architecture, Building and Civil Engineering, Loughborough University, Leicestershire, U.K. \\ *The corresponding author: email gongsheng.huang@cityu.edu.hk; Tel 852-34422408
}

\begin{abstract}
Event-driven optimal control was recently developed for central air-conditioning systems to speed up the response of optimal control to irregular changes in the system optioning conditions. In a time-driven paradigm usually the optimization is carried out with a constant frequency, however the event-driven optimal control triggers optimization actions by events, which will be essentially defined to catch up with the irregular changes. Considering that the occurrence of events should imply the necessity to execute optimization, this paper investigates the necessity of optimization actions, based on which a new method to develop event-driven optimal control law is proposed. This can naturally lead to the establishment of an event-action map. This map indicates that not all the decision variables should be optimized when an event occurs, different from other methods that require optimizing all decision variables. The merits of the new method were also demonstrated using several case studies.
\end{abstract}

Keywords: Real-time optimal control; Event-driven optimization; Energy efficiency; Airconditioning system

\section{Introduction}

Real-time optimal control is an approach to optimize the operation of central air-conditioning (AC) systems and hence improve their energy efficiency without sacrificing indoor thermal comfort (Chua et al. 2011; ASHRAE 2015). It always optimizes the set-points for the local 
controllers in $\mathrm{AC}$ systems in response to the changes in their working condition, including outside weather and indoor thermal conditions (Wang 2009). Real-time optimal control has undergone rapid developments since the 1980s (Cumali 1988). Most research have concentrated on the formulation of optimization problems (Kelman, Ma and Borrelli 2013; Wang and Ma 2008) and the development of advanced optimization techniques, such as evolutionary algorithms (Fong, Handy, and Chow 2009; Ma and Wang 2011) and branch and bound (Chang, Lin, and Lin 2009; Fisk 2014).

In the current real-time optimal control, most current strategies adopts a time-driven paradigm (Kusiak, Li, and Tang 2010; Sun et al. 2013; Martyr et al. 2019), i.e. optimization actions are triggered following a fixed frequency or a scheduled timetable during operation of AC systems. For example, Nassif (2005) developed a bi-objective optimal control strategy for an AC system, in which the supply air and the chilled water supply temperatures were optimized every 30 minutes. However, in the operation of a central AC system, many changes are stochastic and hard to predict, such as those in the weather, load, and occupancy conditions. Being a periodic mechanism in nature, time-driven optimal control (TDOC) may fail to capture those aperiodic changes and thus cannot perform the optimization at the right time (Ma, Matusko, and Borrelli 2015). It may also waste the computational resources when the operating conditions are stable, and no optimization is necessary (but is still performed).

The event-driven paradigm offers an alternative to the time-driven paradigm. Its core idea is that "an action should be triggered by an 'event' that is specified as a well-defined condition on the system state or as a random state transition" (Cassandras 2014). The event-driven approach has been used in sampling (Astrom and Bernhardsson 1999), estimation (Xia, Gupta, and Antsaklis 2016), control (Shen, Yan, and Zhang 2016; Borgers, Dolk, and Heemels 2017) and optimization (Cao 2008), and it is especially attractive when many components are connected over a shared network. For example, in the network control system, using the eventdriven method can be more efficient as information transmission (the action) is triggered only when there is a significant change in the plant output measurements (the event) (Zhu, Xia, and Antsaklis 2014).

The event-driven approach has also gain primary applications in AC systems in recent years. 
It has been used in the adaptive control of AC systems (Dhar, Verma, and Behera 2017), building energy management (Xu et al. 2017), and the optimal control of the air-loop of an AC system (Wu, Jia, and Guan 2015; Jia et al. 2018). In the work of Jia et al. (2018), the set-points of the supply air temperature and air volumes were optimized (the action) when state variables, such as the indoor air temperature, crossed the boundary of a predefined range (the event). A whole central AC system including both water and air loops was considered in Wang et al.'s studies, where events were defined using a knowledge-based method (Wang et al. 2016; Wang et al. 2018) or a data mining approach (2017). All these studies showed that compared with the TDOC, the event-driven optimal control (EDOC) was able to capture the aperiodic changes in the operations of HVAC systems, and simultaneously the computational load of the EDOC can be saved significantly. It should be noted that there are many factors that limit the general application of optimal control for HVAC systems and computational complexity might not be the critical one. However, to perform the optimization at the right time and reduce the computational complexity could improve the cost-to-benefit ratio of implementing the realtime optimal control in HVAC systems.

Despite these merits of EDOC, there are several limitations in these previous studies. First, the knowledge-based or the data mining method are not a systematic way to define events. Tryand-error should be used to access how "good" the defined events are. Second, in these studies a direct map between events and individual decision variables has not been established. When an event occurs, all the decision variables must be optimized. This might not be an efficient method to execute optimization, especially when many variables are needed to be optimized. It is possible that the occurrence of an event might only affect part of the decision variables, and hence for this event only those decision variables that are affected should be optimized.

This paper proposed a study to overcome the abovementioned limitations. Considering that the occurrence of events should imply the necessity to execute optimization, this paper investigates the necessity of optimization actions, based on which a new method to develop event-driven optimal control is proposed. This new method identifies the state transitions that have a critical influence on the performance of the real-time optimal control to establish a mathematical relationship between the variation in the optimal values of a decision variable and its critical state transitions. According to this relationship, an EDOC law is set up and naturally, this 
EDOC law leads to the definition of an event and action space as well as the map between them. The event-action map is used to specify which decision variable should be optimized when an event occurs, which indicates that that not all of the decision variables should be optimized when an event occurs, which is different from a traditional strategy that requires to optimize all decision variables.

Considering the mutual interactions among the decision variables, the event and action spaces are improved through action and/or event merging after analyzing the interaction among the decision variables. Case studies are used to demonstrate the development of the EDOC strategy for a typical central AC system. The performance of the developed strategy is assessed using multiple evaluation indices and compared to that of a TDOC method. It is worth noting that since the proposed method is based on the optimization necessity; it is universal and not limited to the application in AC systems.

This paper is organized as follows. Section 2 illustrates the necessity of optimization actions. Section 3 shows the procedure to establish the event and action spaces for EDOC as well as the map between them. Section 4 presents case studies, including the simulation platform for a typical AC system and the development of the EDOC law. Result analysis and discussion are shown in Section 5. Concluding remarks are presented in Section 6.

\section{Necessity of optimization actions}

Before the illustration of EDOC strategy development method for AC systems, the necessity of optimization actions is firstly introduced, which lays the foundation of the proposed development method. Assume there is a simple system, where only a state variable and a decision variable are involved as shown in Eqn. (1) and (2). The state variable $s$ refers to the uncontrollable operation condition, and the decision variable $v$ is controllable by resetting its value.

$$
\begin{gathered}
F=f(s(t), v) \\
v^{o p t}(t)=\underset{v}{\arg \min } f(s(t), v)
\end{gathered}
$$

During the operation of the system, the state variable $s$ varies in the time dimension as shown in Fig. 1. Optimization actions $a c t_{1}, a c t_{2}, \cdots$ are executed in a row to minimize the objective function $f$ in real time by searching the decision variable's optimal values $v_{1}^{o p t}, v_{2}^{o p t}, \ldots$ 
under the state variable's instantaneous values $s_{1}, s_{2}, \cdots$.

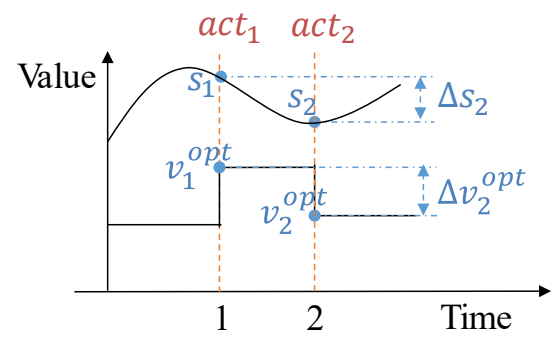

Fig. 1. The state transition and variation of the decision variable's optimal value

Conceptually, an optimization action is necessary when the current value of the decision variable is no longer optimal. Therefore, $a c t_{2}$ is necessary due to non-zero $\Delta v_{2}^{o p t}$, the variation of the searched decision variable's optimal value by $a c t_{2}$ from that by $a c t_{1}$. Furthermore, the larger the deviation, the performed optimization action has the higher necessity. Considering that the variation of the decision variable's optimal values is caused by changes of the state variable, an event will be defined as these state variables' changes leading to an obvious variation of decision variables' optimal values. Events defined in this way will certainly drive optimization actions with a high necessity.

\section{Methodology}

The development method of EDOC strategy for AC systems consists of three steps: 1) identification of critical state transitions, 2) EDOC law, and 3) improving the event-action map.

\section{$\underline{3.1 \text { Identification of critical state transitions for decision variables }}$}

To find state variables' changes leading to an obvious variation of decision variables' optimal values, it is necessary to first identify critical state transitions. These state variables should be selected from weather data (such as outdoor temperature, humidity, solar radiation intensity), operating states of the air-conditioning system (such as the on/off status of components), and/or occupancy conditions. Then, analyze the impact of their variations on selected decision variables.

To this end, time-driven optimal control of AC systems is conducted over a period (from $t=0$ to $t=l$ ). The matrix of the state transitions $M_{s}$ and the matrix of the variation of the decision variables' optimal values and $M_{v}$, as shown in Eqn. (3) and (4), are obtained from operating data. 


$$
M_{s}=\left[\begin{array}{cccc}
\Delta s_{1,1}^{*} & \Delta s_{1,2}^{*} & \ldots & \Delta s_{1, l}^{*} \\
\Delta s_{2,1}^{*} & \Delta s_{2,2}^{*} & \ldots & \Delta s_{2, l}^{*} \\
\vdots & \vdots & \ddots & \vdots \\
\Delta s_{m, 1}^{*} & \Delta s_{m, 2}^{*} & \ldots & \Delta s_{m, l}^{*}
\end{array}\right] \text { with } \Delta s_{j, t}^{*}=\frac{s_{j, t}-s_{j, t-1}}{\max _{t=1 \rightarrow l}\left|s_{j, t}-s_{j, t-1}\right|}, j=1, \cdots, \mathrm{m}, t=1, \cdots, l
$$

$$
M_{v}=\left[\begin{array}{cccc}
\Delta v_{1,1}^{o p t *} & \Delta v_{1,2}^{o p t *} & \ldots & \Delta v_{1, l}^{o p t *} \\
\Delta v_{2,1}^{o p t *} & \Delta v_{2,2}^{o p t *} & \ldots & \Delta v_{2, l}^{o p t *} \\
\vdots & \vdots & \ddots & \vdots \\
\Delta v_{n, 1}^{o p t *} & \Delta v_{n, 2}^{o p t *} & \ldots & \Delta v_{n, l}^{o p t *}
\end{array}\right] \text { with } \Delta v_{i, t}^{*}=\frac{v_{i, t}^{o p t}-v_{i, t-1}^{o p t}}{\max _{t=1 \rightarrow l}\left|v_{i, t}^{o p t}-v_{i, t-1}^{o p t}\right|}, i=1, \cdots, \mathrm{n}, t=1, \cdots, l
$$

160 where $s_{j, t}$ is the value of the $j^{\text {th }}$ state variable at time $t$, and $v_{i, t}^{o p t}$ is the optimal value of the $i^{\text {th }}$ decision variable at time $t$.

Then, the impact of state transitions on variations of decision variables' optimal values are investigated based on the obtained $M_{s}$ and $M_{v}$. Available tools with respect to the identification of critical variables include sensitivity analysis (Saltelli et al. 2004; Tian 2013), feature selection (Guyon and Elisseeff 2003; Zhang and Wen. 2019), and mutual information (May et al. 2008; Kapetanakis, Mangina, and Finn 2017). Due to its simple and low computation cost, the regression-based sensitivity analysis is adopted in this study, which fits a linear regression to the model outputs and uses the regression coefficients as direct measures of sensitivity, as illustrated in studies by Saltelli et al. 2004. The state transitions corresponding to the coefficients with high magnitude are regarded as the critical ones. More details are given in Section 4.3. It is assumed that there are $m_{i}$ critical transitions are identified for the $i^{\text {th }}$ decision variable $v_{i}$, denoted as $\left(\Delta s_{1}^{i}, \cdots, \Delta s_{m_{i}}^{i}\right)$, and $\bar{m}$ critical transitions in total for all decision variables. The map is obtained from the variable selection to link the decision variable and its critical state transitions as shown in Fig. 2.

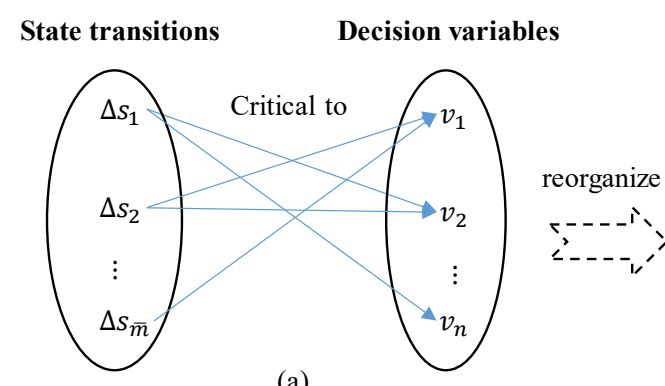

(a)

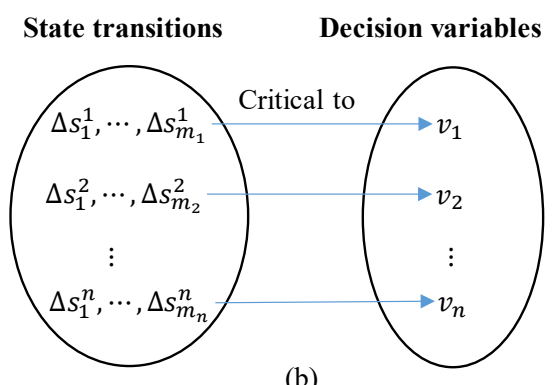

(b)

178 Fig. 2. (a) An initial map to show critical state transitions for decision variables (an example); (b) the improved map to link decision variables and their critical state transitions 


\subsection{EDOC law}

To develop the event-driven paradigm, the important tasks are to build an event/action space, which contains all the events/actions, and a map to link the event and action space all together. The map specifies the appropriate action that should take place when an event occurs. To this end, the mathematical relationship between the variation of each decision variable's optimal value and its critical state transitions should be developed. Regression (Aranda et al. 2012), artificial neural network (ANN) (Kalogirou 2000), and support vector machine (SVM) (Jung, Kim, and Heo 2015) are most techniques commonly used for developing relationship in building energy systems. A simple and explicit regression mode is highly recommended for this study since the event occurrence should be calculated and identified timely. Besides, the relationship developed for event definition is only used to trigger the optimization, not to predict the optimal value of decision variables which are obtained through detailed system models after the trigger. Thus, a simple and explicit regression model can make the immediate identification and satisfy accuracy requirement with less striction.

The mathematical relationship between the critical state transitions and the decision variables is written as

$$
\Delta v_{i}^{o p t}=f^{i}\left(\Delta s_{1}^{i}, \cdots, \Delta s_{m_{i}}^{i}\right)
$$

where $f^{i}$ is a suitable mathematical function, which should be selected according to the data generated by a time-driven optimal control.

Assuming that the optimization of the decision variable $v_{i}(i=1, \cdots, n)$ is necessary when the absolution value of $\Delta v_{i}^{o p t}$ is larger than a predefined threshold $\gamma_{i}$, then an event to trigger the optimization of $v_{i}$ should be defined as

$$
e^{i}:\left|f^{i}\left(\Delta s_{1}^{i}, \cdots, \Delta s_{m_{2}}^{i}\right)\right|>\gamma_{i}
$$

Therefore, the event-driven optimal control (EDOC) law can be written as:

EDOC law: the decision variable $v_{i}, i \in[1, \cdots, n]$ should be optimized at time $t$ if its critical states transition $\left(\Delta s_{1, t}^{i}, \cdots, \Delta s_{m_{i}, t}^{i}\right)$ satisfies $\left|f^{i}\left(\Delta s_{1, t}^{i}, \cdots, \Delta s_{m_{2}, t}^{i}\right)\right|>\gamma_{i}$.

The EDOC law defines the event and action spaces as well as the map between them, as shown in Fig. 3. There are $n$ events and $n$ actions, and each single event triggers one single action. 
It should be noted that the threshold $\gamma_{i}$ in this EDOC law is an important factor to balance the optimization performance and the computational load. Its value should be specified by considering multiple aspects including mainly the sensitivity of the energy consumption of the $\mathrm{AC}$ system to the variation of decision variables, the control precision of control loops for decision variables, and the fluctuation of the system operating state caused by the reset of decision variables.

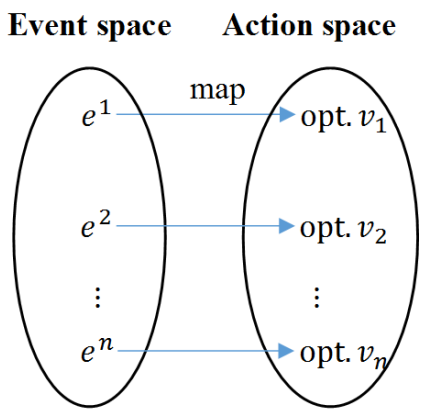

Fig. 3. Event and action space with the map directly from the EDOC law

\subsection{Improve the event-action map}

Fig. 3 indicates that when $e_{i}$ occurs, only $v_{i}$ needs to be optimized. However, in an optimization problem, the global optimal solution can usually be achieved when all decision variables are optimized simultaneously. This is due to the interaction between decision variables which means the optimization of a variable is affected by values taken by other variables ( $\mathrm{Li}$ et al. 2016). Thus, if only the single corresponding decision variable is optimized when an event occurs, the overall performance of the EDOC may be deteriorated. However, if all decision variables are optimized simultaneously when any event occurs, the computation load will increase considerably.

This problem can be avoided by analyzing the correlation among $\Delta v_{i}^{o p t}, i=1, \cdots, n$, and merging the optimization actions of those decision variables with a high correlation coefficient. The merging process is shown in Figure (4a), where $\Delta v_{i}$ and $\Delta v_{j}$ are assumed to be highly correlated. In this case, $v_{j}$ should be optimized when $v_{i}$ is optimized and vice versa. Thus, the optimization of $v_{i}$ and $v_{j}$ should be merged, i.e. whenever $e^{i}$ or $e^{j}$ occurs both $v_{i}$ and $v_{j}$ should be optimized. When the optimization of highly correlated decision variables is merged, the next step is to analyze the independence of the corresponding events and merge events as necessary. For example, if the occurrence of $e^{i}$ will lead to the occurrence of $e^{j}$, 
239 then $e^{i}$ and $e^{j}$ can be merged as $e^{i}$. Note that this merging will not change the definition of $e^{i}$ as shown in Eqn. (6). The final event-action map is shown in Figure (3b), where the links are categorized into two types: Type I where a single event will trigger the optimization of a single decision variable; while in Type II a single event will trigger the optimization of multiple decision variables.

\section{Event space Action space}
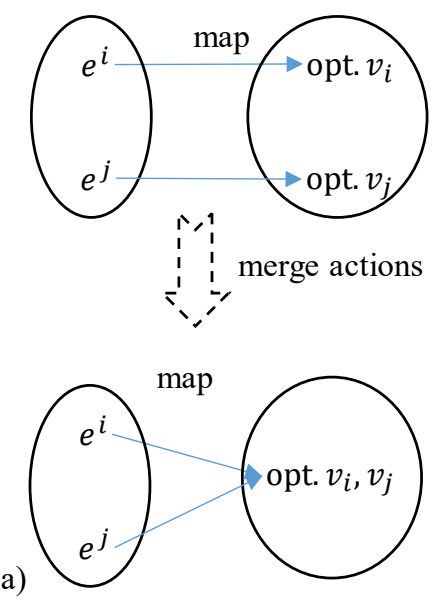

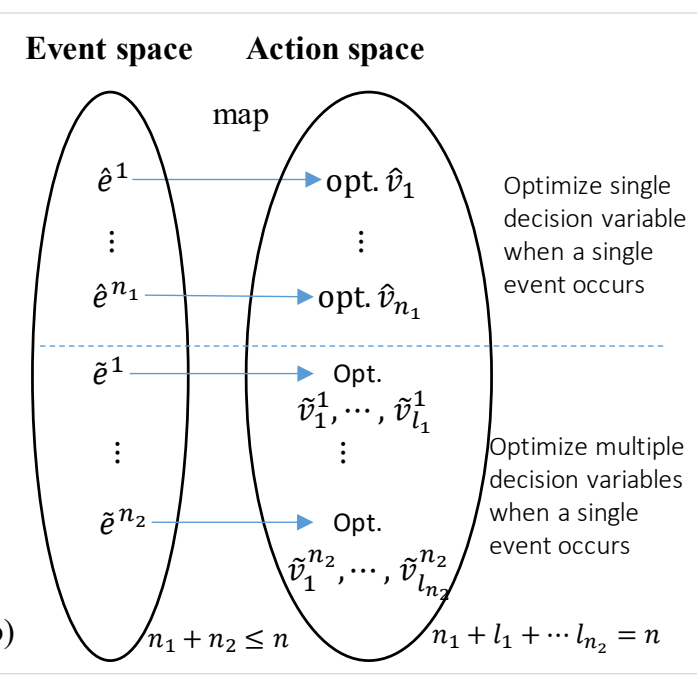

Fig. 4. (a) The process of merging actions; (b) improved map after the analysis of interaction between decision variables

\subsection{Realization of the EDOC strategy}

251 After the event and action spaces, together with their map are established, EDOC can be realized using the flowchart shown in Fig. 5. At each sampling time, all the critical state transitions $\left(\Delta s_{1}, \cdots, \Delta s_{\bar{m}}\right)$ need to be calculated, and hence the corresponding states $\left(s_{1}, \cdots, s_{\bar{m}}\right)$ need to be monitored and their values at each sampling must be recorded. According to the definitions of the events in the event space, the occurrence of each event should be detected. If no event occurs, no optimization should be carried out at this time and therefore the values of the all the decision variables should be maintained unchanged. Otherwise, there is a need to find all the decision variables that should be optimized following the map between the event space and the action space. Next, these variables are optimized using the adopted optimization algorithm and updated to the new optimal values. 


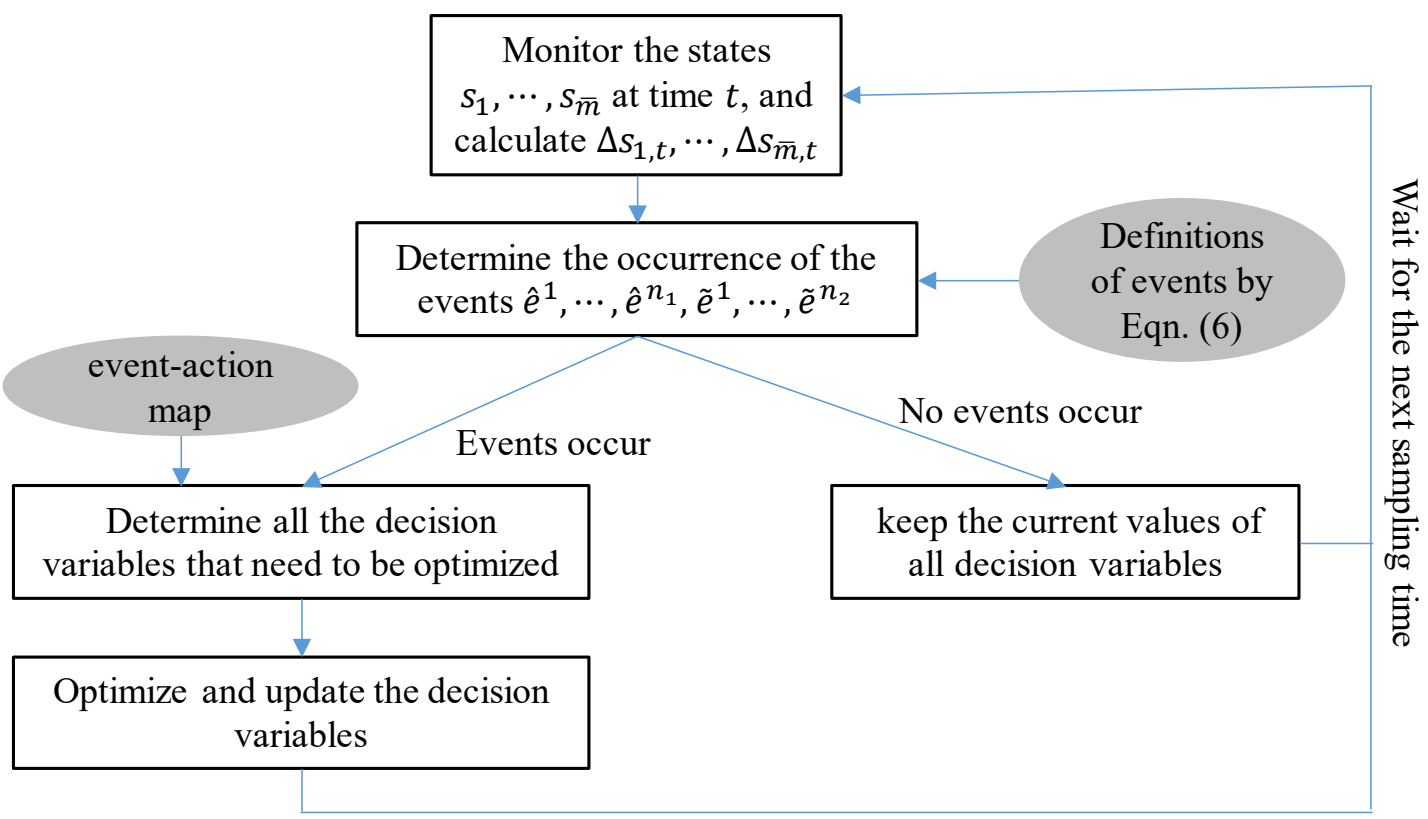

Fig. 5. Visualisation of the developed EDOC strategy 


\section{Case studies}

In this section, the proposed mechanism to develop an EDOC strategy is illustrated through relevant case studies. A typical AC system was first selected, and then its simulation platform was established using TRNSYS and MATLAB. Based on this platform, operating data of AC systems were generated to develop and evaluate the EDO control strategy using the proposed method. The identification of critical state transitions and the establishment of an event-action map are explained below in detail.

\subsection{Simulation platform}

The selected typical air-conditioning system is illustrated in Fig. 6, which has three basic loops: the cooling water $(\mathrm{CW})$, the chilled water (CHW), and the supply air (SA) loops. In the chilled water loop, the chiller is used to generate chilled water, which is delivered by a constant speed pump in the primary side and a variable speed (vs) pump in the secondary side. In the cooling water loop, a constant speed pump is used to cycle the cooling water, which dissipated heat to the ambient. In the supply air loop, the supply air is conditioned by an air-handling unit (AHU) and delivered to zones for cooling. During the operation, the CWS, CHWS, and the SA temperatures were under feedback control to track their preset set-points. The CWS, CHWS and SA temperatures were controlled by adjusting the cooling tower fan frequency, the flow rate of the refrigerant inside the chiller, and the flow rate of the chilled water through the AHU respectively.

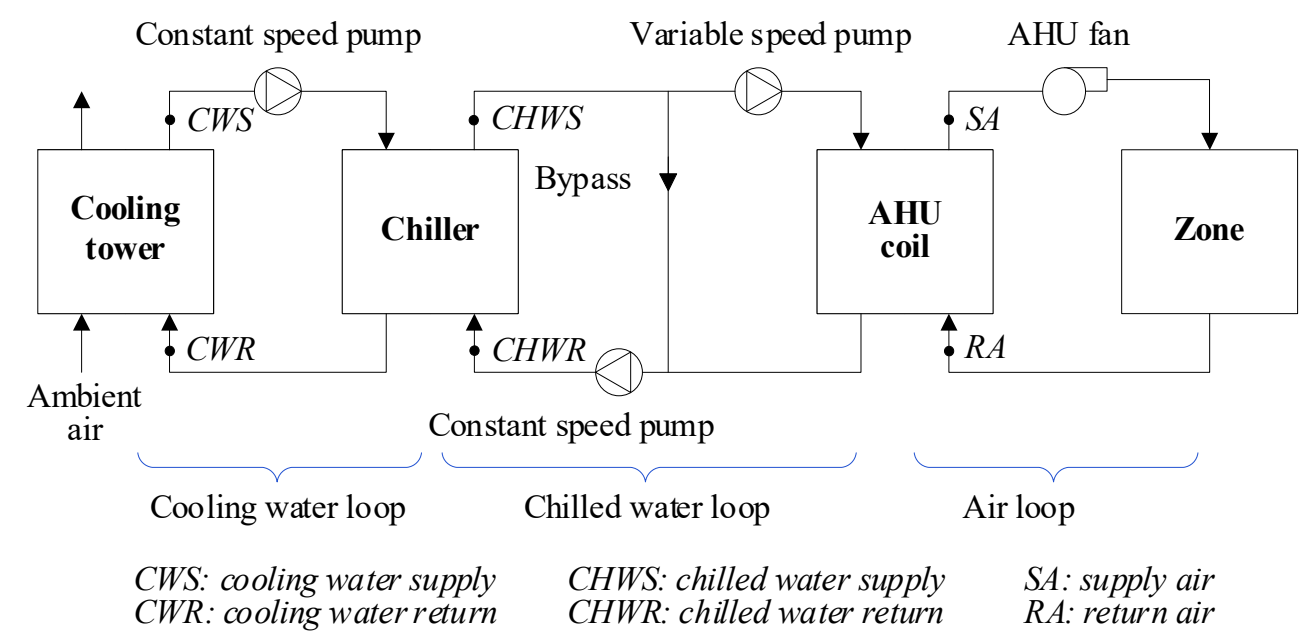

Fig. 6. Schematic of a typical air-conditioning system 
288 TRNSYS was chosen to establish the simulation platform for the AC system because of its proven capability to simulate the dynamics of AC systems. The type numbers of adopted components in TRNSYS and their parameter settings are presented in Table 1. All the component models used in this study are quasi-steady models. Note that external feedback controllers are not used for the control of the CHWS and the SA temperatures since Type 666 and Type 508c themselves assume that the set-points for the CHWS and the SA temperatures to be achieved respectively in their calculations. The performance data within Type 666 was rewritten according to the performance curve of a real chiller (Reform EIR Chiller Screw York YS $781 \mathrm{~kW} / 5.42 \mathrm{COP} /$ Valve) as described in the software DesignBuilder.

Table 1. Parameter settings of components in TRNSYS

\begin{tabular}{lll}
\hline Component & Parameter & Value \\
\hline Chiller & Capacity $(\mathrm{kW})$ & 509 \\
(Type 666) & COP & 6.5 \\
& Chilled water flow rate $(\mathrm{kg} / \mathrm{s})$ & 23.6 \\
& Cooling water flow rate $(\mathrm{kg} / \mathrm{s})$ & 27.8 \\
Cooling tower & Maximum air flow rate $\left(\mathrm{m}^{3} / \mathrm{s}\right)$ & 19.4 \\
(Type 51b) & Rated power $(\mathrm{kW})$ & 5.5 \\
& Cooling water flow rate $(\mathrm{kg} / \mathrm{s})$ & 27.8 \\
& Cooling capacity $(\mathrm{kW})$ & 604 \\
CHW cs pump & Water flowrate $(\mathrm{kg} / \mathrm{s})$ & 23.6 \\
(Type 114) & Head $(\mathrm{m})$ & 15 \\
& Rated power $(\mathrm{kW})$ & 5.1 \\
CW cs pump & Water flowrate $(\mathrm{kg} / \mathrm{s})$ & 27.8 \\
(Type 114) & Head $(\mathrm{m})$ & 15 \\
& Rated power $(\mathrm{kW})$ & 6 \\
CHW vs pump & Water flowrate $(\mathrm{kg} / \mathrm{s})$ & 23.6 \\
(Type 110) & Head $(\mathrm{m})$ & 30 \\
Coil (Type 508c) & Rated power $(\mathrm{kW})$ & 10.4 \\
Fan (Type 111b) & Rated air flow rate $(\mathrm{kg} / \mathrm{s})$ & 0.15 \\
(Type 23) & Rated power $(\mathrm{kW})$ & 37.3 \\
\hline & Gain constant & 40 \\
& Integral time $(\mathrm{hr})$ & 0.1 \\
\hline
\end{tabular}

300 Hong Kong weather for 2003 was selected as the operating environment of the AC system 301 taken from the Hong Kong Observatory. The measured load profiles from a commercial building was used in the simulation. This commercial building had three cooling seasons: 
spring, summer, and autumn as was recorded by the building energy management system (BEMS),. The weather and load data of six days of each season; spring (April), summer (August), and autumn (October) were used as the training condition to develop the event-driven optimal control strategy (see below Section 4.3). The data of a day of each season; spring (April), summer (August), and autumn (October) were used as the test condition to evaluate the developed strategy, and the results are presented in Section 5. Basic information on weather and load data is shown in Table 2.

Table 2. Weather and load condition

\begin{tabular}{|c|c|c|c|c|c|c|c|}
\hline \multirow{2}{*}{ Item } & & \multicolumn{3}{|c|}{ Strategy development } & \multicolumn{3}{|c|}{ Strategy evaluation } \\
\hline & & Spring & Summer & Autumn & Spring & Summer & Autumn \\
\hline Db temp. & Max. & 22.5 & 32.0 & 28.3 & 22.5 & 30.7 & 27.3 \\
\hline \multirow[t]{2}{*}{$\left({ }^{\circ} \mathrm{C}\right)$} & Min. & 18.4 & 25.9 & 21.6 & 19.8 & 26.9 & 22.1 \\
\hline & Mean & 19.8 & 28.8 & 24.7 & 21.1 & 28.8 & 24.6 \\
\hline Wb temp. & Max. & 21.5 & 27.7 & 22.6 & 21.5 & 27.5 & 19.7 \\
\hline \multirow[t]{2}{*}{$\left({ }^{\circ} \mathrm{C}\right)$} & Min. & 16.9 & 24.6 & 14.7 & 18.8 & 25.1 & 16.2 \\
\hline & Mean & 18.9 & 26.5 & 18.8 & 20.3 & 26.2 & 17.8 \\
\hline Cooling load & Max. & 216 & 462 & 344 & 216 & 446 & 294 \\
\hline \multirow[t]{2}{*}{$(\mathrm{kW})$} & Min. & 88 & 94 & 93 & 95 & 105 & 95 \\
\hline & Mean & 135 & 264 & 198 & 161 & 290 & 203 \\
\hline
\end{tabular}

\section{$\underline{4.2 \text { Formulation of real-time optimal control }}$}

Due to no energy storage involved, the real-time optimal control was used to do the static minimization of instantaneous power consumption of the whole system with respect to decision variables at single time point (ASHRAE 2015). The objective function is given by Eqn. (7) and the decision variables were these three set-points for the CWS, CHWS and SA temperature controls.

$$
P_{t o t}=P_{c h}+P_{c t}+P_{c h w, c s}+P_{c h w, v s}+P_{c w, c s}+P_{f a n}
$$

where $P_{t o t}$ is the instantaneous power of the total system; while $P_{c h}, P_{c t}, P_{c h w, c s}, P_{c h w, v s}$, $P_{c w, c s}$, and $P_{f a n}$ are the power of the chiller, cooling tower, constant speed pump for chilled water, variable speed pump for the chilled water, and constant speed pump for the cooling water, and the fan of AHU respectively. The real-time optimization is then formulated as shown in Eqn. (8):

$$
\left[T_{c w, s t}^{o p t}(t), T_{c h w, s t}^{o p t}(t), T_{s a, s t}^{o p t}(t)\right]=\underset{T_{c w, s t}, T_{c h w, s t}, T_{s a, s t}}{\arg \min } P_{t o t}(t)
$$


where $T_{c w, s t}, T_{c h w, s t}$, and $T_{s a, s t}$ denote the set-points for the CWS, CHWS and SA

327

328

329

330

331

332

temperature respectively; while $T_{c w, s t}^{o p t}, T_{c h w, s t}^{o p t}$, and $T_{s a, s t}^{o p t}$ denote their optimal values.

The constraints of this optimization problem were shown in Eqns. (9)-(10). The inequalities in Eqn. (9) indicated that the values of the three decision variables were constrained by lower and upper operating boundaries. The inequalities in Eqn. (10) were used to prevent the system instability from large set-point changes. The operating boundaries of three decision variables are given below in Table 3 .

$$
\begin{gathered}
\left\{\begin{array}{c}
T_{c w, s t}^{L} \leq T_{c w, s t} \leq T_{c w, s t}^{U} \\
T_{c h w, s t}^{L} \leq T_{c h w, s t} \leq T_{c h w, s t}^{U} \\
T_{s a, s t}^{L} \leq T_{a i r, s t} \leq T_{s a, s t}^{U}
\end{array}\right. \\
\left\{\begin{array}{c}
\left|T_{c w, s t}(t)-T_{c w, s t}(t-1)\right| \leq \Delta T_{c w, l m} \\
\left|T_{c h w, s t}(t)-T_{c h w, s t}(t-1)\right| \leq \Delta T_{c h w, l m} \\
\left|T_{s a, s t}(t)-T_{c w, s a}(t-1)\right| \leq \Delta T_{s a, l m}
\end{array}\right.
\end{gathered}
$$

where $T_{c w, s t}^{L} / T_{c w, s t}^{U}, T_{c h w, s t}^{L} / T_{c h w, s t}^{U}$, and $T_{s a, s t}^{L} / T_{s a, s t}^{U}$ are the lower/upper boundaries of $T_{c w, s t}, T_{c h w, s t}$, and $T_{s a, s t}$ respectively, and $\Delta T_{c w, l m}, \Delta T_{c h w, l m}$, and $\Delta T_{s a, l m}$ are the limitation of set-point changes of $T_{c w, s t}, T_{c h w, s t}$, and $T_{s a, s t}$ respectively.

Table 3. Operating boundaries of decision variables

\begin{tabular}{ll}
\hline Boundary & Value $\left({ }^{\circ} \mathrm{C}\right)$ \\
\hline$\left[T_{c w, s t}^{L}, T_{c w, s t}^{U}\right]$ & {$\left[T_{w b}+1.5,35\right]$} \\
{$\left[T_{c h w, s t}^{L}, T_{c h w, s t}^{U}\right]$} & {$[4,10]$} \\
{$\left[T_{s a, s t}^{L}, T_{s a, s t}^{U}\right]$} & {$[12,16]$} \\
\hline
\end{tabular}

To obtain the relationship between these three temperatures set-points and the total power, performance models for all power-consuming components are required. Here it is assumed that these temperature set-points are well tracked, and thus the relationships between the power and these three temperatures can be used directly. For the chillers, the DOE2 model described in the study by Hydeman and Gillespie 2002, was adopted to predict the power and the CHWS temperature for a given evaporator cooling load and the CHWS temperature. For the cooling towers, the simplified cooling tower model in the paper by Lebrun et al. 2004 was used, which was able to predict the required air flow rate for a given CWS temperature and thus the power 
of the fans used in the cooling tower. The performance model of the AHU coils was developed based on Type 508c in TRNSYS. The coil model was able to predict the required CHW flow rate and the outlet water temperature given the CHWS temperature, the SA temperature and humidity. The power of variable speed pumps/fans was described using a cubic polynomial of fluid flowrate. The power of constant speed pumps/fans was constant and was set at their rated power. All undetermined parameters of performance models were identified using the components operating data in TRNSYS to make these performance models consistent with the component in TRNSYS.

The optimization algorithm was programmed using MATLAB and connected with TRNSYS using Type 155, an interface provided in TRNSYS to link TRNSYS and MATLAB. At each optimization time, a comprehensive search was adopted to find the optimal solutions. In this method, the total power $P_{\text {tot }}$ was calculated when the decision variables varied from their lower to the upper boundary at a step change of $0.1^{\circ} \mathrm{C}$. Here the step change of $0.1^{\circ} \mathrm{C}$ was selected as relatively accurate solutions could be obtained and be used for the comparison studies in Section 5. The combination of the decision variables that made $P_{\text {tot }}$ the minimum was the optimal solution $\left[T_{c w, s t}^{o p t}, T_{c h w, s t}^{o p t}, T_{s a, s t}^{o p t}\right]$. The optimal solution was then transferred to TRNSYS and used as the set-points for the control of the CWS, CHWS and SA temperature respectively.

It should be noted that the optimization formulation for a real chiller plant with multiple chillers, cooling towers and AHUs might be much more complex than the one described by Eq. (8), especially when discrete and continuous decision variables are incorporated (Dullinger, Struckl, and Kozek. 2018). However, since the proposed EDOC strategy aims to build a map between events and actions and use existing techniques to solve optimization problems, it can be applied to any chiller plants.

\subsection{Development of the EDOC strategy}

The TDOC for the AC system was carried out under the training condition (18 days) with three different frequencies: every 30, 60 and 120 min (Wang and Ma 2008). In each optimization action, three temperature set-points were optimized simultaneously. Note the constraints in Eq. (10) were not considered in this study so that the operating data could fully reflect the influence 
of state transitions on the variation of the optimal value of decision variables. In this way, operating data for 18 days were obtained.

\subsubsection{Identification of critical state transitions for decision variables}

Since the operation of a chiller plant is mainly affected by its external operating conditions, which can be characterized by the ambient dry-bulb temperature $T_{d b}$, the ambient wet-bulb temperature $T_{w b}$, and the part-load ratio (PLR) of the chiller plant (ASHRAE 2015), they were considered as the states that had a potentially impact on the optimization of the chiller plant. Therefore, critical state transitions were identified from the variation of these three state variables for the optimization of the three decision variables $T_{c w, s t}, T_{c h w, s t}$, and $T_{s a, s t}$.

As illustrated in the theory of sensitivity analysis (Saltelli et al. 2004), the linear regression coefficients of the input and output of a system can be used to measure the importance of input on the output variables. Therefore, taking the state transitions as the input and the variation of decision variables' optimal values as the output, regression coefficients matrix $C_{r}$ in Eqn.(11) is used as the indicator to identify critical state transitions in this case study.

$$
M_{v}=C_{r} \cdot M_{S}
$$

Fitting Eqn. (11) with the operating data, the regression coefficient matrix $C_{r}$ for the whole year (overall of the three seasons) was obtained and shown in Eqn. (12).

$$
\left[\begin{array}{c}
\Delta T_{c w, s t}^{o p t, *} \\
\Delta T_{c h w, s t}^{o p t, *} \\
\Delta T_{s a, s t}^{o p t, *}
\end{array}\right]=\left[\begin{array}{ccc}
-0.027 & \mathbf{0 . 9 3 2} & \mathbf{0 . 4 9 5} \\
0.019 & 0.010 & \mathbf{- 0 . 9 9 7} \\
0.023 & 0.06 & \mathbf{- 1 . 0 1 6}
\end{array}\right] \cdot\left[\begin{array}{c}
\Delta T_{d b}{ }^{*} \\
\Delta T_{w b}{ }^{*} \\
\Delta P L R^{*}
\end{array}\right]
$$

The coefficients with high magnitude in each row are in bold in the matrix, where the magnitude being larger than 0.1 was considered "high" as an example, and their corresponding state transitions were regarded as the critical ones. Other state transitions were then considered insignificant and their impacts were ignored. Therefore, three findings from the four coefficient matrixes are as follows:

(1) The change in the ambient dry-bulb temperature $\Delta T_{d b}$ had a little impact on the variations of all these three decision variables; however, the wet-bulb temperature $\Delta T_{w b}$ had a significant impact on all these three decision variables.

(2) For the CWS temperature set-point $T_{c w, s t}$, its critical state transitions were $\Delta T_{w b}$ and $\triangle P L R$. 
(3) For the CHWS temperature set-point $T_{c h w, s t}$ and the SA temperature set-point $T_{s a, s t}$, their critical state transition was $\triangle P L R$ only.

The map linking the decision variables and their critical state transitions are shown in Fig. 7.

\section{State transitions Decision variables}

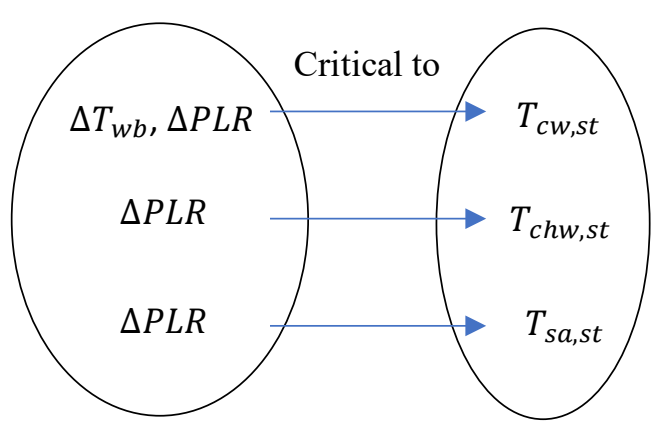

Fig. 7. The decision variable and its critical state transitions

\subsubsection{EDOC law}

The linear functions shown in Eqn. (13) were adopted to describe the relationship between the variation of the decision variable optimal values and their critical state transitions:

$$
\left\{\begin{array}{c}
\Delta T_{c w, s t}^{o p t}=a_{1} \cdot \Delta T_{w b}+b_{1} \cdot \Delta P L R \\
\Delta T_{c h w, s t}^{o p t}=b_{2} \cdot \Delta P L R \\
\Delta T_{s a, s t}^{o p t}=b_{3} \cdot \Delta P L R
\end{array}\right.
$$

By fitting the operation data, into Eqn. (13) the linear models for the whole year were obtained as presented in Eqn. (14), where the coefficient of determination $\left(R^{2}\right)$ was also given. As can be seen in Eqn. (14), $R^{2}$ of the linear was equal or greater than 0.9 , which demonstrated that the variations in the optimal values of the decision variables had a good linearity relationship with their critical state transitions.

$$
\left\{\begin{array}{cc}
\Delta T_{c w, s t}^{o p t}=0.92 \cdot \Delta T_{w b}+1.62 \cdot \Delta P L R & \left(R^{2}=0.92\right) \\
\Delta T_{c h w, s t}^{o p t}=-6.71 \cdot \Delta P L R & \left(R^{2}=0.91\right) \\
\Delta T_{s a, s t}^{o p t}=-3.41 \cdot \Delta P L R & \left(R^{2}=0.90\right)
\end{array}\right.
$$

To determine the threshold of the $\gamma_{i}$ in Eqn. (6), the sensitivity of the total power to the variations of the three set-points $\left(T_{c w, s t}, T_{c h w, s t}, T_{s a, s t}\right)$ close to their optimal values was analyzed. Using the simulation platform, the optimal values of these three set-points were firstly calculated under multiple operating conditions where $P L R$ was varied from 0.2 to 0.9 and $T_{w b}$ from $18^{\circ} \mathrm{C}$ to $27^{\circ} \mathrm{C}$. The instantaneous power $P$ of the $\mathrm{AC}$ system under these 
multiple operating conditions was simulated with these three temperature set-points varying close to their optimal values. The results are summarized in Figure 8, where the horizontal axis $\delta_{T}$ indicate the deviation of the current temperature set-point $T_{s t}$ from its optimal value $T_{s t}^{o p t}$ as shown in Eqn. (15); while the vertical axis $\delta_{P} \%$ is the change rate of the current instantaneous power $P$ compared with the optimal instantaneous power $P^{\text {opt }}$, as shown in Eqn. (16).

$$
\begin{aligned}
\delta_{T} & =T_{s t}-T_{s t}^{o p t} \\
\delta_{P} \% & =\left(P-P^{o p t}\right) / P^{o p t}
\end{aligned}
$$

It can be seen from Figure 8 that the instantaneous power close to the optimum was not very sensitive to the variations of these three temperatures. In detail, when the set-points deviated from the optimal values up to at least $0.4^{\circ} \mathrm{C}$, the total instantaneous power was changed by $0.5 \%$ from the optimal value. The 'flat' optimum indicated that it was not necessary to optimize the system when any one of the three set-points deviated slightly from its optimal value (Braun 1989). Meanwhile, large values of $\delta_{T}$ should not be selected as the threshold for $\gamma_{i}$ in Eqn. (6) because a large change in the set-points might cause the system to become unstable. In previous studies of the time-driven optimal control of the AC system [13, 32], set-point changes were usually constrained within 0.4 or $0.5^{\circ} \mathrm{C}$. Therefore, considering the result of sensitivity study and the system stability, $0.4^{\circ} \mathrm{C}$ was selected for all the three set-points. In this way, three events were defined as shown below in Eqn. (17). Thus, the event-action map was established as presented in Figure (9a).

$$
\left\{\begin{array}{c}
e^{1}:\left|0.92 \cdot \Delta T_{w b}+1.62 \cdot \Delta P L R\right| \geq 0.4 \\
e^{2}:|6.27 \cdot \Delta P L R| \geq 0.4 \\
e^{3}:|3.23 \cdot \Delta P L R| \geq 0.4
\end{array}\right.
$$

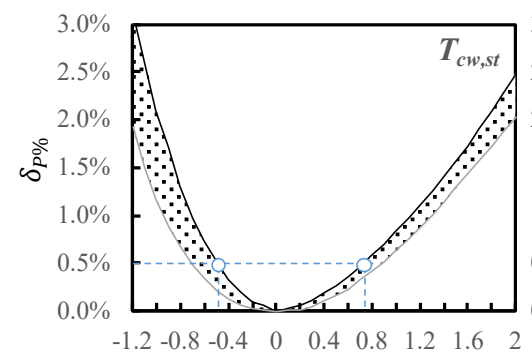

Fig. 8. Sensitivity of the instantaneous power of the AC system to the three temperatures near the optimal value 


\subsubsection{Improve the event-action map}

To improve the event-action map, the interaction between decision variables were analyzed using simulation. To this end, four cases of the TDOC of the AC system were simulated, namely Base case, Case 1, Case 2, and Case 3, which are described in Table 4, where the fixed values are set according to the design guideline of ASHRAE Handbook (2015). In the base case, three decision variables are optimized simultaneously; while in other cases only two decision variables are optimized and the other is remained unchanged. A summer day was selected as an example for the detailed analysis.
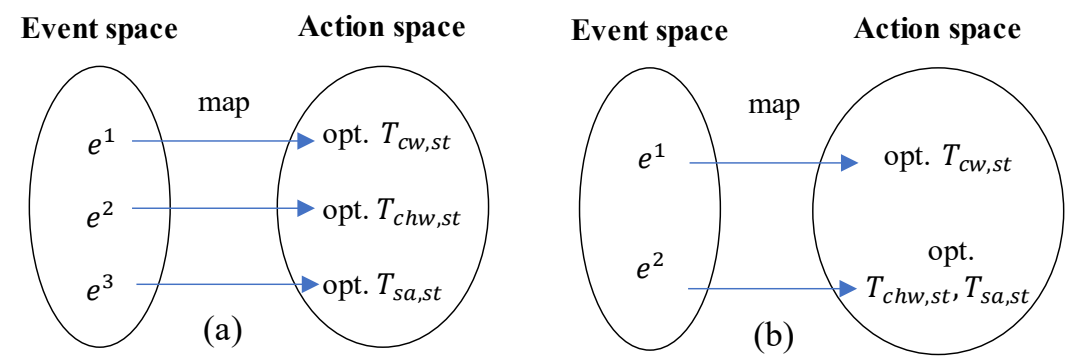

Fig. 9. (a) The event-action map based on the EDOC law; (b) the improved map after

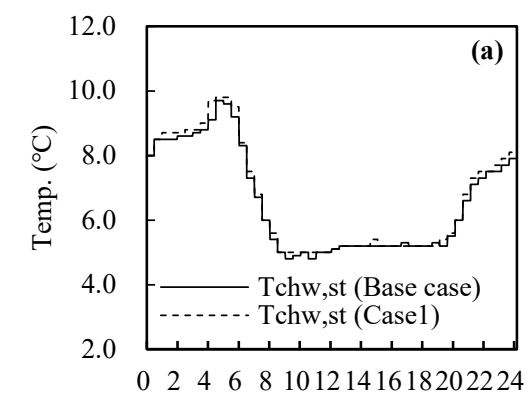
merging events and actions
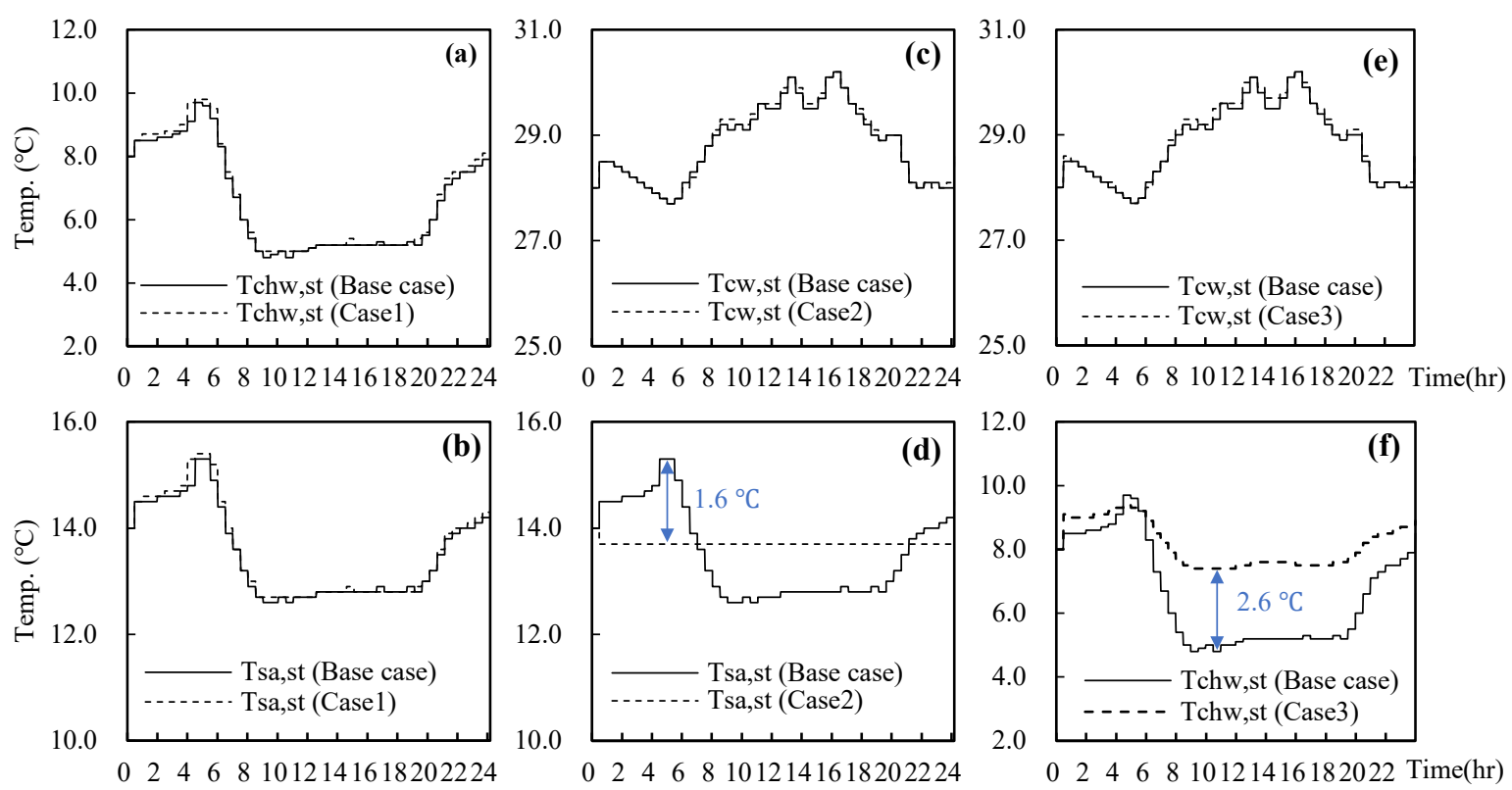

Fig. 10. Profiles of the three temperatures' optimal values for the research of decision 
Table 4. Case information for the research of decision variable interaction

\begin{tabular}{cccc}
\hline Case No. & Optimize & Fixed & Impact $^{*}$ \\
\hline Base case & $T_{c w, s t}, T_{c h w, s t}, T_{s a, s t}$ & - & - \\
Case 1 & $T_{c h w, s t}, T_{s a, s t}$ & $T_{c w, s t}=30^{\circ} \mathrm{C}$ & $T_{c w, s t}$ on $T_{c h w, s t}, T_{s a, s t}$ \\
Case 2 & $T_{c w, s t}, T_{s a, s t}$ & $T_{c h w, s t}=7^{\circ} \mathrm{C}$ & $T_{c h w, s t}$ on $T_{c w, s t}, T_{s a, s t}$ \\
Case 3 & $T_{c w, s t}, T_{c h w, s t}$ & $T_{s a, s t}=15^{\circ} \mathrm{C}$ & $T_{s a, s t}$ on $T_{c w, s t}, T_{c h w, s t}$ \\
\hline
\end{tabular}

478

479

480

481

482

483

${ }^{*}$ : Because $T_{c w, s t}$ in Case 1 was not optimized while it was optimized in the Base case, the comparison between Case 1 and the Base case can help to observe the impact of the optimization of $T_{c w, s t}$ on the optimization of $T_{c h w, s t}$ and $T_{s a, s t}$. If the optimal values of $T_{c h w, s t}$ and $T_{s a, s t}$ in the Base case were consistent with those in Case 1 , it can be concluded that the optimization of $T_{c w, s t}$ had a little impact on the optimization $T_{c h w, s t}$ and $T_{s a, s t}$.

The optimal values of the decision variables in the four cases are presented in Fig. 10. Fig. (10a) and (10b) compared Case 1 (without optimization of $T_{c w}$ ) with the Based case, which showed that the profiles of $T_{c h w, s t}^{o p t}$ and $T_{s a, s t}^{o p t}$ in Case 1 were consistent with that in the Base case. This indicated that the optimization of $T_{c w, s t}$ might not significantly have an impact on the optimization of $T_{c h w, s t}$ and $T_{s a, s t}$. Fig. (10c) and (10d) compared Case 2 (without optimization of $T_{c h w, s t}$ ) with the Base case, and Fig. (10e) and (10f) compared Case 3 (without optimization of $T_{s a, s t}$ ) with the Base case. Fig. (10c) and (10e) showed that the profiles of $T_{c w, s t}^{o p t}$ in Case 2 and Case 3 were consistent with that in the Base case. It demonstrates that the optimization of $T_{c h w, s t}$ and $T_{s a, s t}$ might not have a significant impact on the optimization of $T_{c w, s t}$. Fig. (10d) and (10f) showed that $T_{s a, s t}^{o p t}$ in Case 2 and $T_{c h w, s t}^{o p t}$ in Case 3 were largely deviated from that in the Base case by up to $1.6{ }^{\circ} \mathrm{C}$ and $2.6{ }^{\circ} \mathrm{C}$. This large deviation indicated that $T_{c h w, s t}$ and $T_{s a, s t}$ interact with each other, and thus the two temperatures should be optimized simultaneously. In this way, the event-action map in Figure (9a) should be improved as the one shown in Fig. (9b) by merging $e^{2}$ and $e^{3}$ as $e^{2}$.

\subsection{Optimal control algorithm}

Based on the determined event/action space and the event-action map, the algorithm of the event-driven optimal control for the AC system was developed and its flowchart is shown in Fig. 11. After starting the operation of the AC system, $T_{c w, s t}, T_{c h w, s t}$ and $T_{s a, s t}$ were initially optimized to make sure that these three temperatures were optimal at the beginning and the detection of the events could begin. Then, the ambient wet-bulb temperature $T_{w b}$ and the part-load ratio of the chiller $P L R$ at each sampling period (e.g. every minute) were 
monitored and recorded to detect the occurrence of the events $e^{1}$ and $e^{2}$. To guarantee the operation stability, a time interval $\Delta \Gamma$ between two adjacent optimization actions for the same decision variable should be greater than the time constant of the whole system. Due to the static models adopted and immediate transient responses assumed in the simulation platform, 10minute was used here for minimum time interval for frequent optimization actions. When $e^{1}$ or $e^{2}$ was detected to occur and $\Delta \Gamma \geq 10 \mathrm{~min}, T_{c w, s t}$ or $T_{c h w, s t}$ and $T_{s a, s t}$ should be optimized and their set-points updated accordingly.

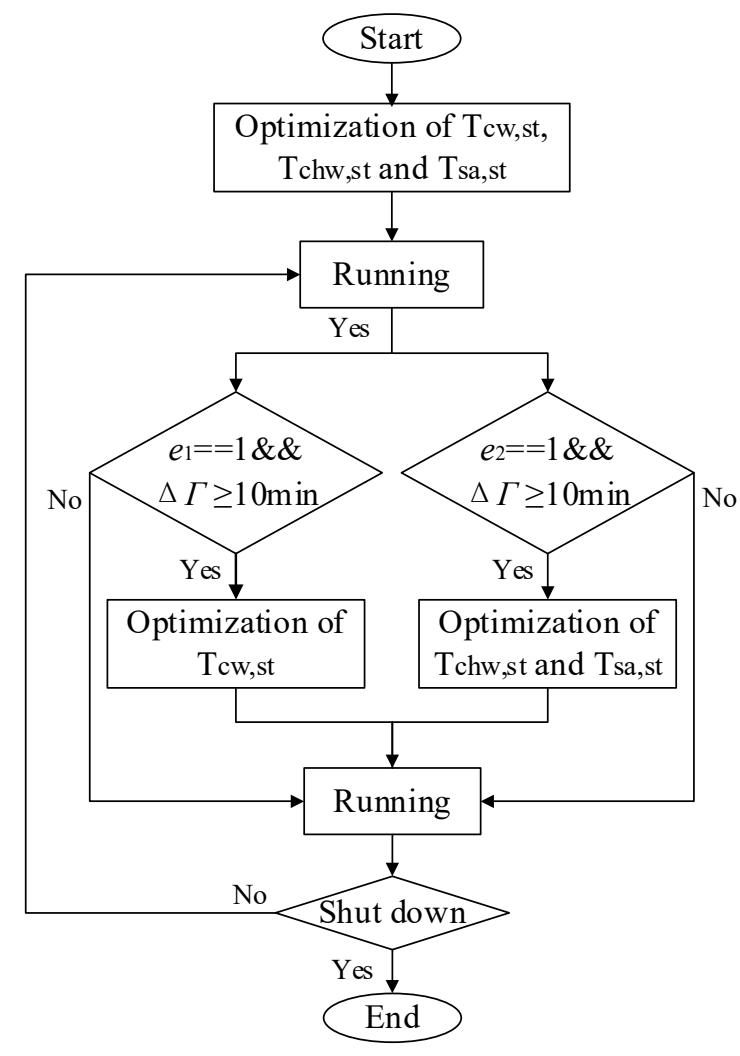

Fig. 11. The flowchart of the event-driven optimal control for the AC system

\section{Results and Discussions}

To evaluate the developed EDOC algorithm, the operation of the AC system was simulated under three strategies: operation without optimal control, operation with time-driven (every $30 \mathrm{~min}$ ) optimal control, and the developed strategy. In the operation without optimal control, these three set-points were fixed at the specific values as listed in Table 5. In the operation with time-driven and event-driven optimal control, the constraints in Eqn. (10) were considered where values of $\Delta T_{c w, l m}, \Delta T_{c h w, l m}$, and $\Delta T_{s a, l m}$ were $0.5^{\circ} \mathrm{C}$ to prevent a large change in setpoints. The operating environments included a spring, a summer, and an autumn day as shown in Table 2. 
Table 5. Fixed values of the three temperatures

\begin{tabular}{lccc}
\hline Item & Spring $\left({ }^{\circ} \mathrm{C}\right)$ & Summer $\left({ }^{\circ} \mathrm{C}\right)$ & Autumn $\left({ }^{\circ} \mathrm{C}\right)$ \\
\hline$T_{c w, s t}$ & 26 & 30 & 26 \\
$T_{c h w, s t}$ & & 7 & \\
$T_{s a, s t}$ & & 15 & \\
\hline
\end{tabular}

526

\subsection{Optimization necessity}

528 As previously mentioned, the development method of EDOC strategy presented in this study aims to achieve high necessity of optimization actions. To test whether it has been achieved, two evaluation indices are proposed, the action distance $d_{v_{i}, t}$ and average distance $\bar{d}$.

The action distance $d_{v_{i}, t}$ was used to evaluate the necessity of the single performed optimization action at time instant $t$ for the decision variable $v_{i}$. As defined in Eqn. (18), it was the absolute variation of one decision variable's optimal value between two adjacent optimization actions. The larger the action distance, the higher the necessity of the action for $v_{i}$

$$
d_{v_{i}, t}=\left|v_{i, t}^{o p t}-v_{i, t-1}^{o p t}\right|=\left|\Delta v_{i, t}^{o p t}\right|
$$

where $v_{i, t}^{o p t}$ is the $i^{t h}$ decision variable's optimal value at time instant $t$.

The average distance $\bar{d}$ was used to evaluate the overall optimization necessity of an optimization strategy considering all performed optimization actions and all decision variables. It was calculated by Eqn. (19). The larger the index, the higher the overall optimization necessity is.

$$
\bar{d}=\frac{\sum_{t=1}^{N_{o p t}} \sum_{v_{i}=v_{1}}^{n v_{t}}\left|d_{v_{i}, t}\right|}{N_{o p t}^{n v}}
$$

where $N_{o p t}^{n v}$ is the weighted optimization times, $N_{o p t}$ is the number of optimization actions, $n v_{t}$ is the number of optimized decision variables in the $t^{t h}$ optimization action, $N_{o p t}^{n v}$ is the optimization times weighted by the number of the optimized decision variables in each optimization action and was calculated by the following equation:

$$
N_{o p t}^{n v}=\sum_{t=1}^{N_{o p t}} n v_{t}
$$

The action distance and average distance of TDOC and EDOC are calculated and presented in 
Figure 12 and Table 6. Figure 12 gave the frequency distribution histogram of all action distance in the two optimal control strategies. It can be observed that the action distances for the three optimized temperatures in the TDOC were mainly inside the range of $\left[0^{\circ} \mathrm{C}, 0.1^{\circ} \mathrm{C}\right]$; while most of action distances in the EDOC had a large value such as $0.4^{\circ} \mathrm{C}$ or $0.5^{\circ} \mathrm{C}$. The average distance of the TDOC was $0.11^{\circ} \mathrm{C}$ in spring, $0.15^{\circ} \mathrm{C}$ in summer, and $0.14^{\circ} \mathrm{C}$ in autumn; while in the EDOC it was $0.39^{\circ} \mathrm{C}$ in spring, $0.33^{\circ} \mathrm{C}$ in summer, and $0.35^{\circ} \mathrm{C}$ in autumn. The large distance of the EDOC indicated that the defined events by the proposed development method had effectively avoided unnecessary optimization actions and thus increased the overall optimization necessity compared with the TDOC.

\subsection{Computation load}

The computation load of the two optimal control strategies was evaluated using the computation time $\tau_{\text {opt }}$. It refers to the time required by the optimization method to search for optimal solutions. The computation time consumed by TDOC was $11.82 \mathrm{~s}$ in spring, $11.18 \mathrm{~s}$ summer, and 11.92s and autumn as shown in Table 6. Compared with the TDOC, the relative computation time saving $r_{C S}$ was $83.9 \%$ in spring, $85.2 \%$ in summer, and $75.9 \%$ in autumn. The great reduction in computation load in the EDOC strategy was caused by the removal of unnecessary optimization actions and the decrease of the search dimension of the optimizations. The search dimension of optimizations in EDOC control was decreased by the event-action map. With the map, not all three temperatures were optimized in each optimization action, so that the computation time for each optimization action was reduced. For example, the average computation time for each optimization action and each temperature in the summer day was $\tau_{\text {opt }} / N_{\text {opt }}^{n v}=0.078 \mathrm{~s}$ in the TDOC and $0.033 \mathrm{~s}$ in the EDOC.

\subsection{Energy performance}

The energy performance of TDOC and EDOC strategies was compared with the operation without optimization. As shown in Table 6, the daily energy consumption of the operation without optimization was $976 \mathrm{kWh}$ in spring, $1747 \mathrm{kWh}$ in summer and $1135 \mathrm{kWh}$ in autumn. The relative energy saving $r_{E S}$ of TDOC was $4.4 \%$ in spring, $7.5 \%$ in summer, and $4.8 \%$ in autumn, and that of the EDOC was $4.2 \%$ in spring, $7.4 \%$ in summer, and $4.8 \%$ in autumn respectively. Therefore, the EDOC strategy achieved nearly the same energy performance as that of the TDOC with a frequency of every 30 minutes. The reason for the effective energy 
saving of the EDOC can be explained in Figure 13 and 14 when the summer day is taken as an example. Figure 13 presents the operating conditions of the summer day, and Figure 14 compares the optimal values of the three set-points from the two optimal control strategies. It is observed that the profiles of $T_{c w, s t}^{o p t}, T_{c h w, s t}^{o p t}$ and $T_{s a, s t}^{o p t}$ in the two optimal control methods were consistent. Besides, the EDOC responded more quickly to the rapid change of the operation conditions. For example, the cooling load during 6:00 to 9:00 am increased rapidly as shown in Figure 13. Figure (14b) and (14c) showed that optimization actions of the EDOC were carried out more frequently and $T_{c h w, s t}^{o p t}$ and $T_{s a, s t}^{o p t}$ changed more quickly than that in the TDOC during the same period. In this way, energy saving effect of the EDOC strategy developed by the proposed method can be guaranteed although weighted optimization times are greatly reduced compared with the TDOC.

Table 6. Evaluation of event-driven optimization strategy

\begin{tabular}{|c|c|c|c|c|c|c|}
\hline Case & $\begin{array}{l}\text { Average distance } \\
\left({ }^{\circ} \mathrm{C}\right) \bar{d}\end{array}$ & $\begin{array}{c}\text { Opt. times } \\
N_{\text {opt }}^{n v}\end{array}$ & $\begin{array}{l}\text { Computation } \\
\text { time (s) } \tau_{\text {opt }}\end{array}$ & $\begin{array}{l}\text { Computation } \\
\text { saving } r_{C S}\end{array}$ & $\begin{array}{l}\text { Energy }(\mathrm{kWh}) \\
E_{\text {ben }} \text { or } E_{\text {opt }}\end{array}$ & $\begin{array}{c}\text { Energy saving } \\
r_{E S}\end{array}$ \\
\hline \multicolumn{7}{|l|}{ Spring } \\
\hline No opt. & - & - & - & - & 976 & - \\
\hline TDOC & 0.11 & 144 & 11.82 & - & 933 & $4.4 \%$ \\
\hline EDOC & 0.39 & 27 & 1.90 & $83.9 \%$ & 935 & $4.2 \%$ \\
\hline \multicolumn{7}{|l|}{ Summer } \\
\hline No opt. & - & - & - & - & 1747 & - \\
\hline TDOC & 0.15 & 144 & 11.18 & - & 1616 & $7.5 \%$ \\
\hline EDOC & 0.33 & 51 & 1.66 & $85.2 \%$ & 1617 & $7.4 \%$ \\
\hline \multicolumn{7}{|l|}{ Autumn } \\
\hline No opt. & - & - & - & - & 1135 & - \\
\hline TDOC & 0.14 & 144 & 11.92 & - & 1080 & $4.8 \%$ \\
\hline EDOC & 0.35 & 42 & 2.87 & $75.9 \%$ & 1081 & $4.8 \%$ \\
\hline
\end{tabular}



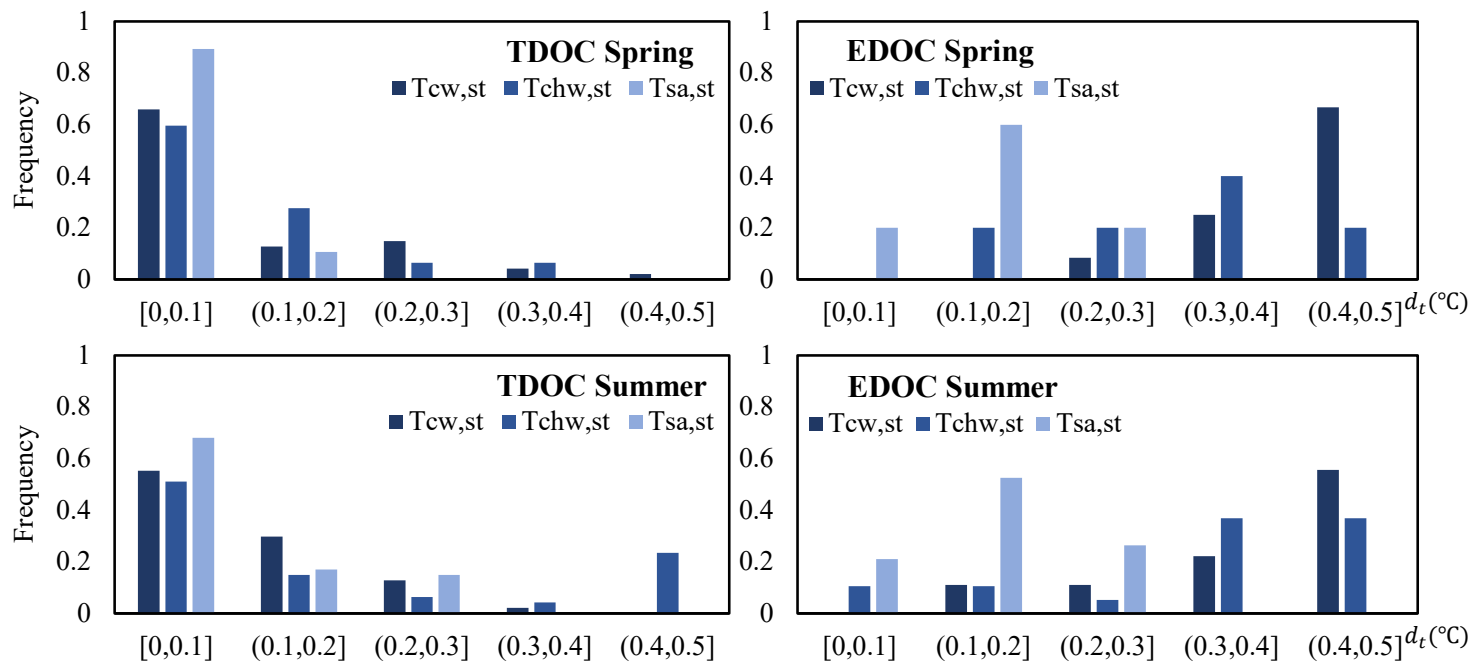

\section{EDOC Summer}

$\square$ Tcw,st $\square$ Tchw,st $\varpi$ Tsa,st
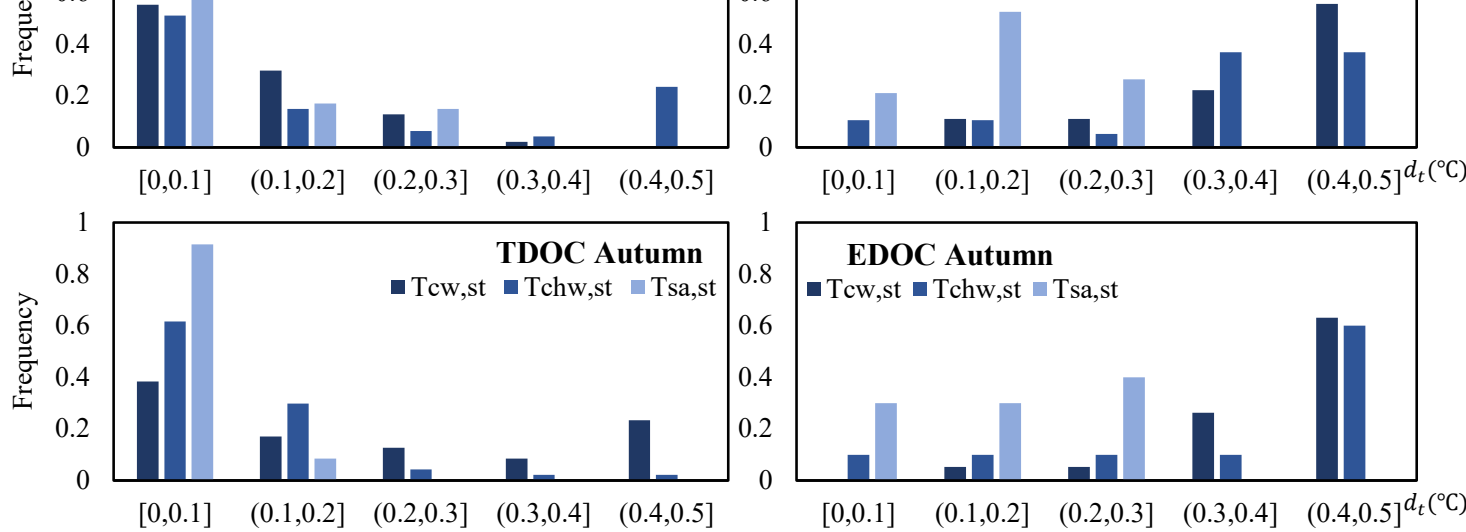

Fig. 12. Frequency distribution histograms of the optimization distance in TDOC and EDOC.

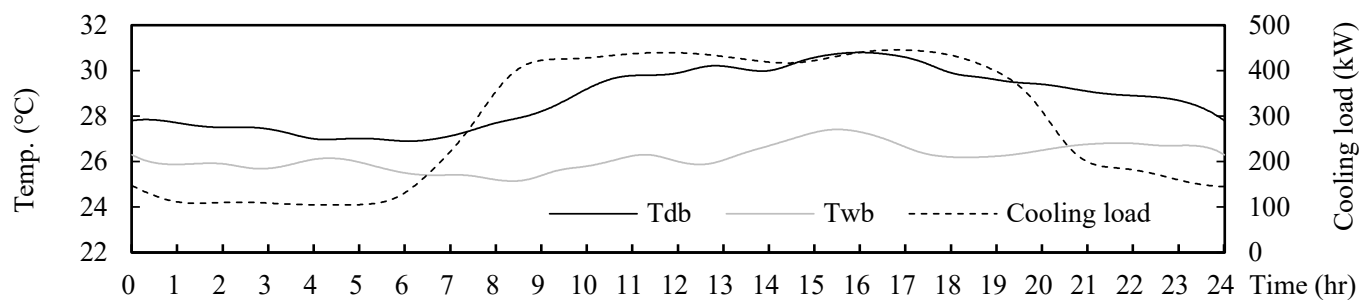

Fig. 13. Profiles of operating conditions in the summer day
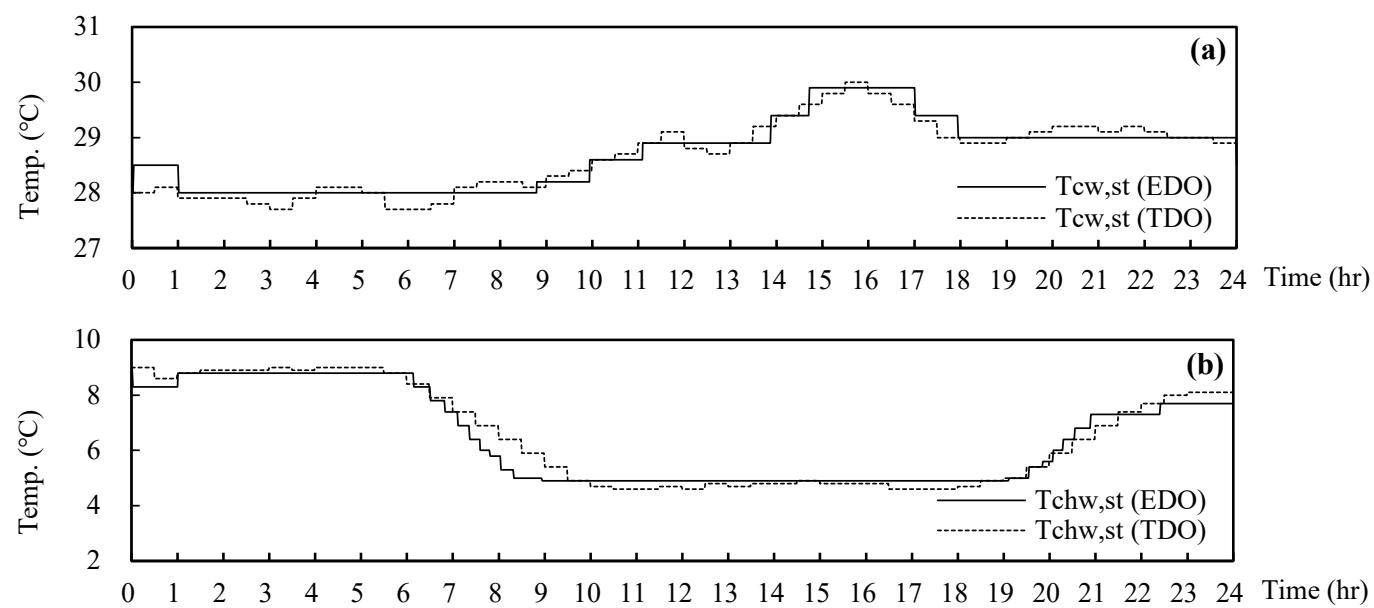


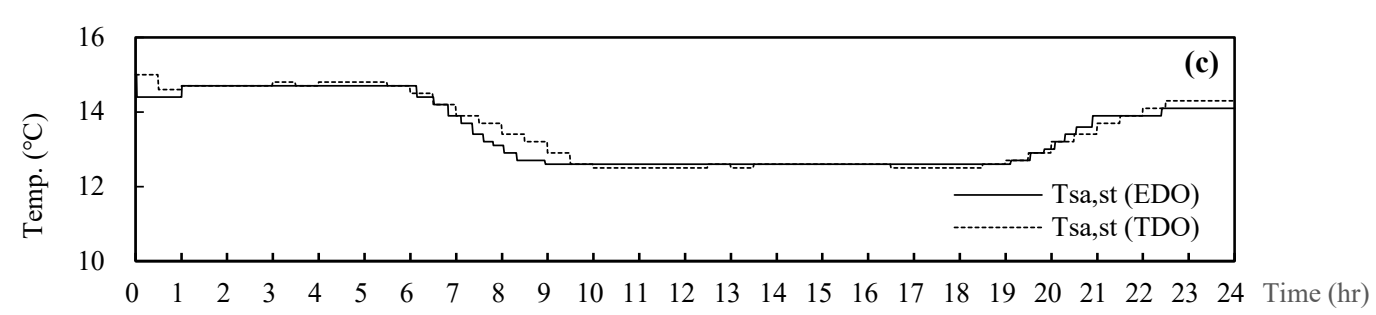

Fig. 14. Profiles of the three temperatures' optimal values in the summer day

\section{Conclusions}

This study has addressed the necessity of optimization actions in the real-time optimal control of air-conditioning systems, based on which a new method to develop event-driven optimal control is proposed. In the development method, the event/action space and the event-action map can be determined in a natural and systematic way, and not all the decision variables need to be optimized for a given event. The proposed method has been applied to a typical airconditioning system, in which one event have been defined for the single optimization of a decision variable, the set-point for the cooling water supply temperature, and the other event for the combined optimization of other two decision variables including the set-points for the chilled water supply and the supply air temperatures. Compared with the operation with no optimization, the event-driven optimal control strategy can reduce 4.2-7.4\% energy use, which is comparable to that of the time-driven optimal control (4.4-7.5\%). Compared with the timedriven optimal control, the average distance in the event-driven optimization was $0.33-0.39^{\circ} \mathrm{C}$, being 2.2-3.5 times of the time-driven control $\left(0.11-0.15^{\circ} \mathrm{C}\right)$. Therefore, the developed eventdriven optimization can achieve a significant computation load reduction up to $75.9-85.2 \%$ compared with the time-driven optimization (with a frequency of 30 mins) without scarifying the energy-saving potential.

In future work, the proposed method will be applied to a typical central air-conditioning system under different climates and for different types of buildings in order to identify generic events for air-conditioning systems. At the same time, the definition of events based on internal state transitions, such as on/off for major components, faults, and operators' intervention, will also be considered.

\section{Acknowledgment}

The research work presented in this paper was supported by a grant from the Research Grants 


\section{Reference}

635

Aranda, A., G. Ferreira, M. Mainar-Toledo, S. Scarpellini, and E. L. Sastresa. 2012. "Multiple Regression Models to Predict the Annual Energy Consumption in the Spanish Banking Sector.” Energy and Buildings 49: 380387.

ASHRAE. 2015. ASHRAE Handbook - HVAC Applications (SI Edition). Atlanta, GA: ASHRAE.

Åström, K.J., and B. Bernhardsson. 1999. “Comparison of Periodic and Event based Sampling for First-order Stochastic Systems.” IFAC Proceedings Volumes 32: 5006-5011.

Borgers, D. P., V. S. Dolk, W. Heemels. 2017. "Riccati-based Design of Event-triggered Controllers for Linear Systems with Delays.” IEEE Transactions on Automatic Control 63: 174-188.

Braun, J. E., S.A. Clein, J. W. Mitchell, and W.A. Beckman. 1989. "Methodologies for Optimal Control of Chilled-water Systems without Storage.” ASHRAE transactions 95: 952-662.

Cao, X. R. 2008. "Stochastic Learning and Optimization - a Sensitivity-based Approach." IFAC Proceedings Volumes 41: 3480-3492.

Cassandras, C.G. 2014. "The Event-driven Paradigm for Control, Communication and Optimization.” Journal of Control and Decision 1: 3-17.

Chang, Y.C., F.A. Lin, and C.H. Lin. 2005. “Optimal Chiller Sequencing by Branch and Bound Method for Saving Energy." Energy Conversion and Management 46: 2158-2172.

Cumali, Z. 1988. "Global Optimization of HVAC System Operations in Real Time.” ASHRAE Transactions 94: 1729-1744.

Dhar, N. K., N. K. Verma, and L. Behera. 2017. “Adaptive Critic-based Event-triggered Control for HVAC System.” IEEE Transactions on Industrial Informatics 14: 178-188.

Dullinger, C., W. Struckl, and M. Kozek. 2018. “A General Approach for Mixed-integer Predictive Control of HVAC Systems Using MILP.” Applied Thermal Engineering 128: 1646-1659.

Fisk, D.J. 2014. “Optimising Heating System Structure Using Exergy Branch and Bound.” Building Services Engineering Research \& Technology 35: 321-333.

Guyon, I., and A. Elisseeff. 2003. "An Introduction to Variable and Feature Selection." Journal of Machine Learning Research 3: 1157-1182.

Hydeman, M., and K. L. Gillespie. 2002. "Tools and Techniques to Calibrate Electric Chiller Component Models.” ASHRAE transactions 108: 733-741.

Jia, Q. S., J. Wu, Z. Wu, and X. Guan. 2018. "Event-based HVAC Control-A Complexity-based Approach." IEEE Transactions on Automation Science and Engineering 15: 1909-1919.

Jung, H. C., J. S. Kim, and H. Heo. 2015. "Prediction of Building Energy Consumption using an Improved Real Coded Genetic Algorithm based Least Squares Support Vector Machine Approach.” Energy and Buildings 90: 76-84.

Kalogirou, S.A. 2000. “Applications of Artificial Neural-Networks for energy systems.” Applied energy 67: 1735. 
Kapetanakis, D. S., E. Mangina, and D. P. Finn. 2017. "Input Variable Selection for Thermal Load Predictive Models of Commercial Buildings." Energy and Buildings 137: 13-26.

Kelman, A., Y. Ma, and F. Borrelli. 2013. "Analysis of Local Optima in Predictive Control for Energy Efficient Buildings." Journal of Building Performance Simulation 6: 236-255.

Kusiak, A., M.Y. Li, and F. Tang. 2010. "Modeling and optimization of HVAC energy consumption." Applied Energy 87: 3092-3102.

Lebrun, J., C.A. Silva, F. Trebilcock, and E. Winandy. 2004. "Simplified Models for Direct and Indirect Contact Cooling Towers and Evaporative Condensers." Building Services Engineering Research and Technology 25: 25-31.

Li K., M. N. Omidvar, K. Deb, X. Yao. 2016. "Variable Interaction in Multi-objective Optimization Problems." In: Handl J., Hart E., Lewis P., López-Ibáñez M., Ochoa G., Paechter B. (eds) Parallel Problem Solving from Nature - PPSN XIV. PPSN 2016. Lecture Notes in Computer Science, vol. 9921. Springer, Cham.

Ma, Y., J. Matusko, and F. Borrelli. 2015. "Stochastic Model Predictive Control for Building HVAC Systems: Complexity and Conservatism." IEEE Trans. Contr. Sys. Techn. 23: 101-116.

Ma, Z. J., and S. W. Wang. 2011. "Supervisory and Optimal Control of Central Chiller Plants using Simplified Adaptive Models and Genetic Algorithm." Applied Energy 88: 198-211.

Martyr, R., J. Moriarty, and C. Beck. 2019. "Optimal Control of a Commercial Building's Thermostatic Load for Off-peak Demand Response.” Journal of Building Performance Simulation 12: 580-594.

May, R. J., H. R. Maier, G. C. Dandy, and T. G. Fernando. 2008. "Non-linear Variable Selection for Artificial Neural Networks using Partial Mutual Information." Environmental Modelling \& Software 23: 1312-1326.

Nassif N., S. Kajl, and R. Sabourin. 2005. "Optimization of HVAC Control System Strategy Using Two-objective Genetic Algorithm.” HVAC\&R Research 11: 459-486.

Omidvar, M.N., X. Li, Y. Mei, and X. Yao. 2013. "Cooperative Co-evolution with Differential Grouping for Large Scale Optimization." IEEE Transactions on evolutionary computation 18: 378-393.

Saltelli, A., S. Tarantola, F. Campolongo, and M. Ratto. 2004. "Sensitivity Analysis in Practice: A Guide to Assessing Scientific Models.” John Wiley \& Sons Ltd, Chichester, West Sussex, England.

Shen, M., S. Yan, and G. Zhang. 2016. "A new Approach to Event-triggered Static Output Feedback Control of Networked Control Systems." ISA transactions 65: 468-474.

Sun, Y. 2017. "On the Analysis of Interaction between Decision Variables." PhD thesis, Department of Mechanical Engineering, the University of Melbourne.

Sun, Y.J., G.S. Huang, Z.W. Li, and S.W. Wang. 2013. "Multiplexed Optimization for Complex Air Conditioning Systems." Building and Environment 65: 99-108.

Tian, W. 2013. "A Review of Sensitivity Analysis Methods in Building Energy Analysis." Renewable and sustainable energy reviews 20: 411-419.

Waibel, C., G. Mavromatidis, R. Evins., and J. Carmeliet. 2019. "A Comparison of Building Energy Optimization Problems and Mathematical Test Functions Using Static Fitness Landscape Analysis." Journal of Building Performance Simulation 12: 789-811.

Wang, J., Q. S. Jia, G. S. Huang, and Y. J. Sun. 2018. "Event-driven Optimal Control of Central Air-conditioning Systems: Event-space Establishment.” Science and Technology for the Built Environment 24: 839-849. 
Wang, J. Q., G. S. Huang, Y. J. Sun, and X. P. Liu. 2016. "Event-driven Optimization of Complex HVAC Systems.” Energy and Buildings 133: 79-87.

Wang, J. Q., P. Zhou, G. S. Huang, and W. Wang. 2017. “A Data Mining Approach to Discover Critical Events for Event-Driven Optimization in Building Air Conditioning Systems.” Energy Procedia 143: 251-257.

Wang, S. W. 2009. Intelligent Buildings and Building Automation. Spon Press, Taylor \& Francis, London and New York.

Wang, S. W., and Z. J. Ma. 2008. "Supervisory and Optimal Control of Building HVAC Systems: a Review." HVAC\&R Research 14: 3-32.

Wu, Z., Q. S. Jia, and X. Guan. 2015. "Optimal Control of Multi-room HVAC System: An Event-based Approach.” IEEE Transactions on Control Systems Technology 24: 662-669.

Xia, M., V. Gupta, and P.J. Antsaklis. 2016. "Networked State Estimation over a Shared Communication Medium." IEEE Transactions on Automatic Control 62: 1729-1741.

Xu, Z., G. Hu, C. J. Spanos, and S. Schiavon. 2017. "PMV-based Event-triggered Mechanism for Building Energy Management under Uncertainties." Energy and Buildings 152: 73-85.

Zhang, L., and J. Wen. 2019. "A Systematic Feature Selection Procedure for Short-term Data-driven Building Energy Forecasting Model Development." Energy and Buildings 183: 428-442.

Zhu, F., M. Xia, and P. J. Antsaklis. 2014. "Passivity Analysis and Passivation of Interconnected Event-triggered 